\title{
Hybrid Model for Fast Solution of Thermal Synchronous Generator With Heat Exchanger
}

\author{
Khaled Aleikish, Madhusudhan Pandey, Thomas Øyvang, Bernt Lie \\ University of South-Eastern Norway, Porsgrunn, Norway, Bernt. Lie@usn.no
}

\begin{abstract}
Overheating of synchronous generators may lead to a shortened generator lifespan, thus strict constraints are imposed on their operation. A common constraint is to restrict the power factor of the generator to lie below, say, 0.86 overexcited. In some recent work, a dynamic thermal model of the generator with cooling heat exchanger has been developed; the idea is that this allows for better monitoring of generator temperature, while relaxing on the power factor constraint. The current model is only valid for an ideal case of constant heat capacity. In this work, the generator model is extended to allow for temperature dependence in heat capacity of water and air in the heat exchanger model. The consequence of this more realistic model, is that it is no longer possible to find an explicit, analytic solution of the heat exchanger model, and it is now necessary to instead solve numerically a two point boundary value problem for each time step in the differential equation solver.

It is shown that the effect of temperature dependence in the heat capacities has a noticeable effect on the solution of the model. The inclusion of on-line numeric solution of the heat exchanger model does, however increase the computation time of the thermal generator model by a factor of several thousand. Here, we instead study the possibility to solve the numeric heat exchanger model multiple times off-line, and then fit a regression model that gives a correction to the analytic solution. Both linear regression and nonlinear regression (neural network) is considered. Both types of regression models allow for a speed-up in the computation time of the thermal generator model of a factor of around 2000. In the computations, computer language Julia was used, with the DifferentialEquations and the Flux packages.

Keywords: linear regression, nonlinear regression, thermal model, machine learning, surrogate model, hybrid model.
\end{abstract}

\section{Introduction}

\subsection{Background}

Synchronous generators operated at high power factor face high currents, and potential overheating. To protect the generators from overheating, European hydropower generation limits the power factor to the range $[0.85,0.95]$ overexcited, while in Norway the power factor should be less than or equal to 0.86 (Pandey, 2019). It is of interest to allow for more flexible constraints on the power factor to better handle sudden changes in consumption. To do this, it is necessary with good monitoring and control of generator temperatures; this possibility was discussed in (Øyvang, 2018).

\subsection{Previous work}

As a further study of the thermal generator model in $(\varnothing y-$ vang, 2018), $\mathrm{Lie}^{1}$ proposed a slightly more formal model, still assuming constant heat capacity in materials. This model was further studied in (Pandey, 2019); Pandey et al., 2019), where temperature dependences in heat capacities were introduced. Temperature dependence in heat capacities of air and water invalidates the analytic solution of the heat exchanger model, requiring the numeric solution of a static two-point boundary value (TPBV) problem for every time step in the time integration. The numeric solution of the TPBV problem is much slower than evaluating the analytic expressions ( $\mathrm{Lie}^{2}$ ), leading to excessive computation time for model fitting, and on-line uses such as state estimation and advanced control. It is therefore of interest to study the development of simplified models/surrogate models for the heat exchanger. A standard procedure would be to solve the TPBV heat exchanger model multiple times off-line, and then fit a regression model to the generated data. Data for surrogate models do not contain measurement noise per se, so an alternative to a regression model could be table look-up or interpolation. Still, regression models are often simpler, and there may be some "noise" due to inaccuracies in numeric computations. The problem of overfitting the data is relevant for surrogate models.

\subsection{Overview of the paper}

Instead of solving the nonlinear TPBV problem of temperature dependent heat capacities within the time integrator of the thermal synchronous generator model, data-driven surrogate models were developed for the nonlinear heat exchanger model as a correction to the analytic solution. The goal is that this hybrid heat exchanger model consisting in the analytic, ideal solution combined with the ex-

\footnotetext{
${ }^{1}$ Lie, B.: Solution to Project in course FM1015 Modelling of Dynamic Systems at University of South-Eastern Norway, 2018.

${ }^{2}$ Lie, B.: Lecture Notes in course FM1015 Modelling of Dynamic Systems at University of South-Eastern Norway, 2019.
} 


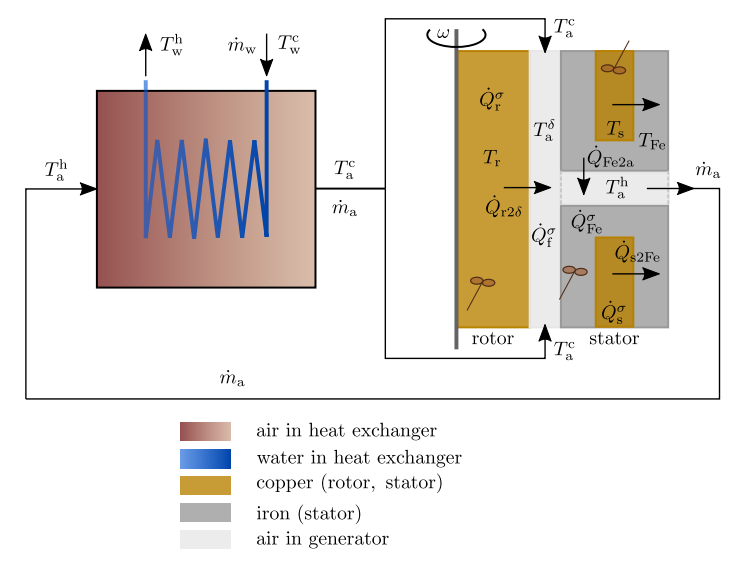

Figure 1. Thermal model of aircooled synchronous generator.

plicit surrogate correction, should give considerably faster solution time for the thermal generator model. Both linear regression and nonlinear regression (neural network) are considered, and validation is used to choose model order/avoid overfitting.

The paper is organized as follows. Section 2 describes the problem that arise from temperature dependence in the heat capacities and the impact on simulation time. Section 3 describes linear regression and the idea of validation, while Section 4 describes nonlinear regression. Results are discussed in Section 5, and some conclusions are given in Section 6.

\section{Solution of the Counter-Current Heat Exchanger Model}

A thermal model of a counter-current heat exchanger was developed in $\mathrm{Lie}^{3}$.

The following explicit/analytic expressions were formed for the effluent temperatures of the tube side $\left(T_{\mathrm{e}}^{\mathrm{t}}\right)$ and the shell side $\left(T_{\mathrm{e}}^{\mathrm{s}}\right)$ :

$$
\begin{gathered}
T_{\mathrm{e}}^{\mathrm{t}}=\frac{N_{\mathrm{St}}^{\triangle} T_{\mathrm{i}}^{\mathrm{t}} \exp \left(-N_{\mathrm{St}}^{\triangle}\right)+N_{\mathrm{St}}^{\mathrm{t}} T_{\mathrm{i}}^{\mathrm{s}}\left(1-\exp \left(-N_{\mathrm{St}}^{\triangle}\right)\right)}{N_{\mathrm{St}}^{\mathrm{t}}-N_{\mathrm{St}}^{\mathrm{s}} \exp \left(-N_{\mathrm{St}}^{\triangle}\right)} \\
T_{\mathrm{e}}^{\mathrm{s}}=\frac{N_{\mathrm{St}}^{\triangle} T_{\mathrm{i}}^{\mathrm{s}}+N_{\mathrm{St}}^{\mathrm{s}} T_{\mathrm{i}}^{\mathrm{t}}\left(1-\exp \left(-N_{\mathrm{St}}^{\triangle}\right)\right)}{N_{\mathrm{St}}^{\mathrm{t}}-N_{\mathrm{St}}^{\mathrm{s}} \exp \left(-N_{\mathrm{St}}^{\triangle}\right)} .
\end{gathered}
$$

Here, $T_{\mathrm{i}}^{\mathrm{t}}$ and $T_{\mathrm{i}}^{\mathrm{s}}$ are the influent temperatures of the heat exchanger. $\quad N_{\mathrm{St}}^{\mathrm{t}}$ and $N_{\mathrm{St}}^{\mathrm{s}}$ are the Stanton numbers of the tube side and the shell side, respectively. Also, $N_{\mathrm{St}}^{\triangle}$ is the difference in Stanton numbers, and is expressed as:

$$
N_{\mathrm{St}}^{\triangle}=N_{\mathrm{St}}^{\mathrm{t}}-N_{\mathrm{St}}^{\mathrm{s}} .
$$

In addition, the temperature profile across the heat exchanger length can be obtained by substituting the explicit expression of $T_{\mathrm{e}}^{\mathrm{s}}$ in the following equations:

\footnotetext{
${ }^{3}$ Lie, B.: Lecture Notes in course FM1015 Modelling of Dynamic Systems at University of South-Eastern Norway, 2019.
}

$$
\begin{aligned}
& T_{\mathrm{t}}(x)=\frac{\left(N_{\mathrm{St}}^{\mathrm{t}} e^{\left(-N_{\mathrm{St}}^{\triangle}\right) x}-N_{\mathrm{St}}^{\mathrm{s}}\right) T_{\mathrm{i}}^{\mathrm{t}}+\left(N_{\mathrm{St}}^{\mathrm{t}}-N_{\mathrm{St}}^{\mathrm{t}} e^{\left(-N_{\mathrm{St}}^{\triangle}\right) x}\right) T_{\mathrm{e}}^{\mathrm{s}}}{N_{\mathrm{St}}^{\triangle}} \\
& T_{\mathrm{S}}(x)=\frac{\left(N_{\mathrm{St}}^{\mathrm{s}} e^{\left(-N_{\mathrm{St}}^{\triangle}\right) x}-N_{\mathrm{St}}^{\mathrm{s}}\right) T_{\mathrm{i}}^{\mathrm{t}}+\left(N_{\mathrm{St}}^{\mathrm{t}}-N_{\mathrm{St}}^{\mathrm{s}} e^{\left(-N_{\mathrm{St}}^{\triangle}\right) x}\right) T_{\mathrm{e}}^{\mathrm{s}}}{N_{\mathrm{St}}^{\triangle}} .
\end{aligned}
$$

The Stanton numbers $N_{\mathrm{St}}^{\mathrm{t}}$ and $N_{\mathrm{St}}^{\mathrm{s}}$ for the tube side and the shell side, respectively, are given as

$$
\begin{aligned}
N_{\mathrm{St}}^{\mathrm{t}} & =\frac{\mathscr{U} A_{\mathrm{x}}}{\hat{c}_{p, t} \dot{m}_{\mathrm{t}}} \\
N_{\mathrm{St}}^{\mathrm{s}} & =\frac{\mathscr{U} A_{\mathrm{x}}}{\hat{c}_{p, \mathrm{~s}} \dot{m}_{\mathrm{s}}},
\end{aligned}
$$

where $\mathscr{U} A_{\mathrm{x}}$ is the heat transfer coefficient, $\hat{c}_{p, \mathrm{t}}$ and $\hat{c}_{p, s}$ are the specific heat capacities at constant pressure, and $\dot{m}_{\mathrm{t}}$ and $\dot{m}_{\mathrm{s}}$ are mass flow rates.

The previous analytic expressions are only valid for an ideal heat exchanger model with constant Stanton numbers. For a more realistic heat exchanger model, specifically, a model with temperature dependence in the heat capacities, a numeric solution is required. In this work, the two-point boundary value problem of the thermal model is solved numerically by utilizing the boundary value problem (BVP) solvers available in the DifferentialEquations package for Julia (Rackauckas and Nie, 2017).

In the thermal model of $\mathrm{Lie}^{4}$, cold air is blown by a fan into the gap between the rotor and the stator, which cools the synchronous generator before returning to the shell side of the counter-current heat exchanger. There, the hot air is cooled by cold water passing in the tube side. In this work, temperature dependence is only considered in the heat capacities of air and water in the heat exchanger part of the model.

Temperature dependence in the heat capacities is often expressed as an empirical power series in $T$ or as a polynomial in $T$ (Murphy, 2020). In this work, polynomials were fitted to the experimental data in (Incropera et al., 2013) and compared with the empirical equations in (McBride et al., 2002). Figure 2 shows the comparison between the specific heat capacities of (Incropera et al., 2013) and the empirical equations of (McBride et al., 2002).

Furthermore, to study the impact of the temperature dependence of the specific heat capacities of air and water on the solution of the counter-current heat exchanger model, the following models are considered:

- Model 1: An ideal heat exchanger model, which is solved using the analytic expressions. The specific heat capacities of air and water are constant and equal $1.15 \mathrm{~kJ} / \mathrm{kg} / \mathrm{K}$ and $4.2 \mathrm{~kJ} / \mathrm{kg} / \mathrm{K}$, respectively.

\footnotetext{
${ }^{4}$ Lie, B.: Solution to Project in course FM1015 Modelling of Dynamic Systems at University of South-Eastern Norway, 2018.
} 

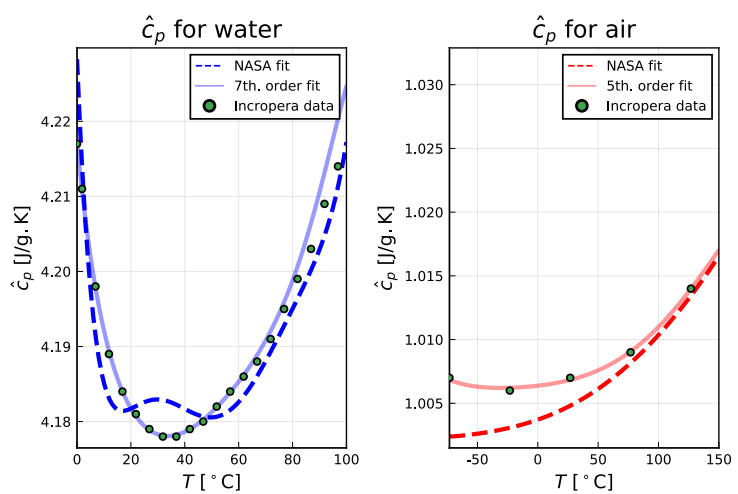

Figure 2. A comparison between the specific heat capacities of (Incropera et al., 2013) and the empirical equations of (McBride et al., 2002).

- Model 2: An ideal heat exchanger model, which is solved using the BVP solvers. The specific heat capacities are the same as in Model 1.

- Model 3: A non-ideal heat exchanger model with $\hat{c}_{p}(T)$, which is solved using the BVP solvers. The specific heat capacities are described by polynomials constructed from the experimental data in Incropera et al. (2013).

Figure 3 shows a comparison between the solution of Model 1 and the solution of Model 3, where the operating conditions are described in Table 1. Also, Table 2 shows the benchmark results (simulation time) for the three models.

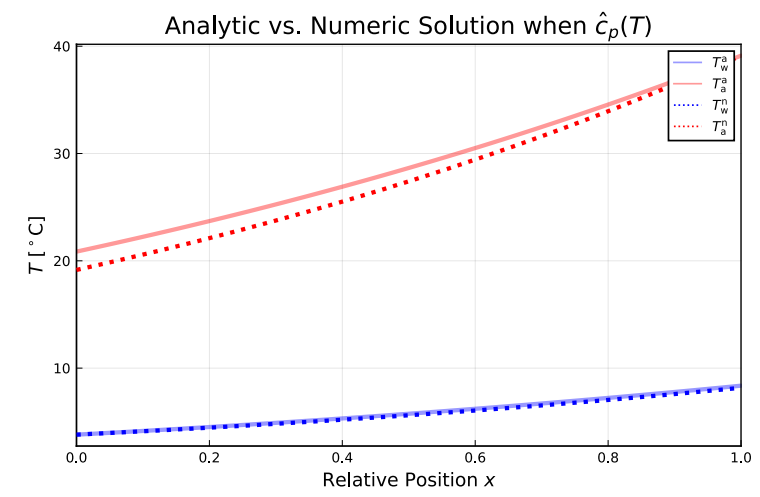

Figure 3. Analytic solution (solid lines) vs. numeric solution (dotted lines) when the specific heat capacities depend on temperature. For the ideal model, the specific heat capacities of air and water are constant, and equals $1.15 \mathrm{~kJ} / \mathrm{kg} / \mathrm{K}$ and $4.2 \mathrm{~kJ} / \mathrm{kg} / \mathrm{K}$, respectively. For the numeric solution, the specific heat capacities are described by polynomials constructed from the experimental data in (Incropera et al., 2013).

Figure 4 shows a comparison between thermal generators model with heat exchanger Model 1 vs Model 3 at the operating conditions. Table 3 shows the benchmark results for the generator model when the three heat exchanger sub-models are used.
Table 1. Operating conditions of the heat exchanger model.

\begin{tabular}{lll}
\hline Symbol & Description & Value \\
\hline \multicolumn{3}{c}{ Inputs $u$} \\
\hline$T_{\mathrm{w}}^{\mathrm{c}}$ & Cold water temperature & $3.8^{\circ} \mathrm{C}$ \\
$T_{\mathrm{a}}^{\mathrm{h}}$ & Hot air temperature & $39.1^{\circ} \mathrm{C}$ \\
$\dot{m}_{\mathrm{w}}$ & Water mass flow rate & $53.9 \mathrm{~kg} / \mathrm{s}$ \\
$\dot{m}_{\mathrm{a}}$ & Air mass flow rate & $49.2 \mathrm{~kg} / \mathrm{s}$ \\
\hline \multicolumn{3}{c}{ Parameters $\theta$} \\
\hline $\mathscr{U} A_{\mathrm{x}}$ & Heat transfer, air to water & $44.46 \mathrm{~kW} / \mathrm{K}$ \\
\hline
\end{tabular}

Table 2. Benchmark results for the heat exchanger model. Model 1 has median time $9 \mu$ s and mean time $10.6 \mu$ s on a given computer; times are scaled to unity at $1^{\text {st }}$ row element.

\begin{tabular}{lcc}
\hline Model & Median time & Mean time \\
\hline Model 1 & 1 & 1 \\
Model 2 & 443 & 451 \\
Model 3 & 2120 & 1940 \\
\hline
\end{tabular}

In this work, the following steps are taken to reduce the simulation time of Model 3 (the case of temperature dependence in the heat capacities):

- First, both Model 1 and Model 3 are solved for a variety of conditions $\left(T_{\mathrm{w}}^{\mathrm{c}}, T_{\mathrm{a}}^{\mathrm{h}}, \dot{m}_{\mathrm{w}}\right.$, and $\left.\dot{m}_{\mathrm{a}}\right)$, and the results are stored in a data matrix.

- Next, a data-driven model is developed by linear/nonlinear regression. Here, the regression model is expressed as a correction term to the analytic expressions.

- Finally, the combination of the regression model and the analytic expressions forms explicit expressions that relate the influent temperature of water/air to the effluent temperature of water/air. Solving explicit expressions is much faster than solving a nonlinear two-point boundary value problem numerically.

\section{Linear regression}

As described in (Gujarati, 2019), given a data set $\left\{y_{i}, x_{1 i}, x_{2 i}, \ldots, x_{k i}\right\}_{i=1}^{n}$ of $n$ observations, a generic linear regression model can be expressed as:

$$
y_{\mathrm{i}}=\beta_{1} x_{1 i}+\beta_{2} x_{2 i}+\ldots+\beta_{k} x_{k i}+\varepsilon_{i}, \quad i=\{1,2,3, \ldots, n\} ;
$$

here, $y$ is called the response variable. $x_{1}, x_{2}, \ldots, x_{k}$ are called the regressor variables. $\beta_{1}, \beta_{2}, \ldots, \beta_{k}$ are the regression coefficients or regression parameters. $\varepsilon$ is called the disturbance term. The subscript $i$ refers to the observation in the data set. Moreover, equation 8 represents a system of equations that can be stacked together and written in matrix notation as:

$$
\mathbf{y}=\mathbf{X} \beta+\varepsilon,
$$

where $\mathbf{y}$ and $\varepsilon$ are $n \times 1$ column vectors, and $\beta$ is a $k \times 1$ column vector. $\mathbf{X}$ is the design matrix of size $n \times k$. In this work, the possible regressors are: 


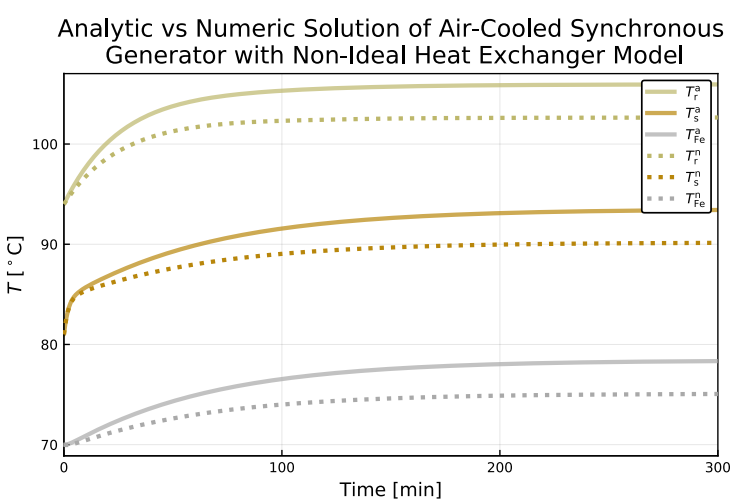

Figure 4. Analytic solution (solid lines) vs. numeric solution (dotted lines) of the thermal generator model when the specific heat capacities depend on temperature.

Table 3. Benchmark results for the thermal model of an aircooled synchronous generator. Model 1 has median time $5.4 \mathrm{~ms}$ and mean time $6.6 \mathrm{~ms}$ on a given computer; times are scaled to unity at $1^{\text {st }}$ row element.

\begin{tabular}{lcc}
\hline Model & Median time & Mean time \\
\hline Model 1 & 1 & 1 \\
Model 2 & 451 & 363 \\
Model 3 & 1820 & 1480 \\
\hline
\end{tabular}

1. The hot water temperature of the analytic solution $\left(T_{\mathrm{w}}^{\mathrm{h}, \mathrm{A}}\right)$

2. The cold air temperature of the analytic solution $\left(T_{\mathrm{a}}^{\mathrm{c}, \mathrm{A}}\right)$

3. Water mass flow rate $\left(\dot{m}_{\mathrm{w}}\right)$

4. Air mass flow rate $\left(\dot{m}_{\mathrm{a}}\right)$

Also, the response variables are the effluent temperatures $\left(T_{\mathrm{w}}^{\mathrm{h}, \mathrm{N}}\right.$ and $\left.T_{\mathrm{a}}^{\mathrm{c}, \mathrm{N}}\right)$ of the numeric solution when $\hat{c}_{p}(T)$. Since the number of response variables $m>1$, then equation 9 is expressed as:

$$
\mathbf{Y}=\mathbf{X} \beta+\varepsilon
$$

here, $\mathbf{Y}$ and $\varepsilon$ are $n \times m$ matrices, and $\beta$ is a matrix of size $k \times m$. $\mathbf{X}$ is a design matrix of size $n \times k$. In this work, the columns of the design matrix are not the regressors themselves, but a polynomial of the regressors, which includes an intercept and cross-product terms. Using the method of ordinary least squares, an estimate of the regression coefficients can be obtained by:

$$
\hat{\beta}=\left(\mathbf{X}^{\top} \mathbf{X}\right)^{-1} \mathbf{X}^{\top} \mathbf{y} .
$$

Then, the regression function is expressed as:

$$
\hat{\mathbf{y}}=\mathbf{X} \hat{\beta}
$$

here, $\hat{\mathbf{y}}$ is the predicted value of $\mathbf{y}$ for a given design matrix. The regression function predicts the effluent temperatures of the non-ideal heat exchanger model given the effluent temperatures of the ideal model.
It is of interest to have a measure of goodness-of-fit, i.e., how well $\hat{y}$ predicts $y$. A simple predictor is the mean $\hat{y}=\bar{y}$, with uncertainty given by the sample variance

$$
\sigma_{y}^{2}=\frac{\sum_{i=1}^{n}\left(y_{i}-\bar{y}\right)^{2}}{n} ;
$$

$\bar{y}=\sum_{i=1}^{n} y_{i} / n$. With predictor $\hat{y}_{i}=\sum_{j=1}^{k} \hat{\beta}_{j} x_{j, i}$, the variance is

$$
\sigma_{\varepsilon}^{2}=\sum_{i=1}^{n}\left(y_{i}-\hat{y}_{i}\right)^{2} / n
$$

with $\varepsilon_{i}=y-\hat{y}_{i}$. Two measures of model quality are the Root Mean Square Error (RMSE) $)^{5}$

$$
\operatorname{RMSE}=\sqrt{\sigma_{\varepsilon}^{2}}=\sigma_{\varepsilon}=\sqrt{\sum_{i=1}^{n}\left(y_{i}-\hat{y}_{i}\right)^{2} / n},
$$

and the Coefficient of Determination $R^{2}$ (Gujarati, 2019)

$$
R^{2}=1-\sigma_{\varepsilon}^{2} / \sigma_{y}^{2} .
$$

For a "perfect" model, $R^{2} \rightarrow 1$, while for a "poor" model (the mean), $R^{2} \rightarrow 0$.

The sample variance $\sigma_{y}^{2}$ is biased because Eq. 13 uses estimated mean $\bar{y}$; estimate $\sigma_{y}^{2}$ has one degree of freedom. The unbiased sample variance $s_{y}^{2}$ is

$$
s_{y}^{2}=\frac{\sum_{i=1}^{n}\left(y_{i}-\bar{y}\right)^{2}}{n-1}=\frac{n}{n-1} \sigma_{y}^{2} .
$$

The prediction error variance uses $k$ estimated parameters $\beta_{j}$, thus has $k$ degrees of freedom, and the corrected estimate $s_{\varepsilon}^{2}$ is

$$
s_{\varepsilon}^{2}=\frac{n}{n-k} \sigma_{\varepsilon}^{2} .
$$

Alternatively to measures RMSE and $R^{2}$, the Standard Error of Estimate (SEE) (Smith, 2015)

$$
\mathrm{SEE}=\sqrt{s_{\varepsilon}^{2}}=s_{\varepsilon}=\sqrt{\sum_{i=1}^{n}\left(y_{i}-\hat{y}_{i}\right)^{2} /(n-k)} .
$$

or the adjusted coefficient of determination $\bar{R}^{2}$ defined as

$$
\bar{R}^{2}=1-s_{\varepsilon}^{2} / s_{y}^{2}=1-\frac{n-1}{n-k}\left(1-R^{2}\right)
$$

are used. RMSE and SEE has the advantage of having the same unit as $y$.

When solving Model 1 and Model 3, the following cases were considered:

- Case 1: Constant mass flow rates and variable influent temperatures. In this case, the regressors are $T_{\mathrm{w}}^{\mathrm{h}, \mathrm{A}}$ and $T_{\mathrm{a}}^{\mathrm{c}, \mathrm{A}}$. Also, the models are solved over the range of $\left(4-30^{\circ} \mathrm{C}\right)$ for $T_{\mathrm{w}}^{\mathrm{c}}$, and over the range of $\left(40-100^{\circ} \mathrm{C}\right)$ for $T_{\mathrm{a}}^{\mathrm{h}}$, while the mass flow rates are kept constant at the values of Table 1 .

${ }^{5}$ https://en.wikipedia.org/w/index.php? title=Mean_squared_error\&oldid=959959954 
- Case 2: Variable influent temperatures and mass flow rates. In this case, the regressors are $\dot{m}_{\mathrm{a}}, \dot{m}_{\mathrm{w}}, T_{\mathrm{w}}^{\mathrm{h}, \mathrm{A}}$, and $T_{\mathrm{a}}^{\mathrm{c}, \mathrm{A}}$. Also, the models are solved over the range of $\left(4-40^{\circ} \mathrm{C}\right)$ for $T_{\mathrm{w}}^{\mathrm{c}}$, and over the range of $(15-$ $\left.130^{\circ} \mathrm{C}\right)$ for $T_{\mathrm{a}}^{\mathrm{h}}$, and over the range of $(15-130 \mathrm{~kg} / \mathrm{s}$ ) for the mass flow rates.

In Fig. 5 and Fig. $6, \overline{\mathrm{R}}^{2}$ and SEE were used to find the order of the polynomial of the design matrix that gives the best fit. Also, it can be observed in the figures that the goodness-of-fit deteriorates due to overfitting as the model order increases beyond a 5 th order for case 1 , and beyond a 6 th order for case 2.

Moreover, Fig. 7 and Fig. 8 show a comparison between the regression models and the numerical solution of the nonlinear two-point boundary value problem for case 1. Also, in Fig. 7, the regression surface and the data points overlap for the 5 th order model, which indicates a good fit. On the other hand, in Fig. 8, the regression surface of the 12th order model does not match the data for some parts, which indicates a bad fit.

Similarly, Fig. 9 and Fig. 10 show a comparison between the regression models and the numerical solution of the nonlinear two-point boundary value problem for case 2. Also, in Fig. 9, the regression surface and the data points overlap for the 6th order model, which indicates a good fit. On the other hand, in Fig. 10, the regression surface of the 12th order model does not match the data for some parts, which indicates a bad fit.
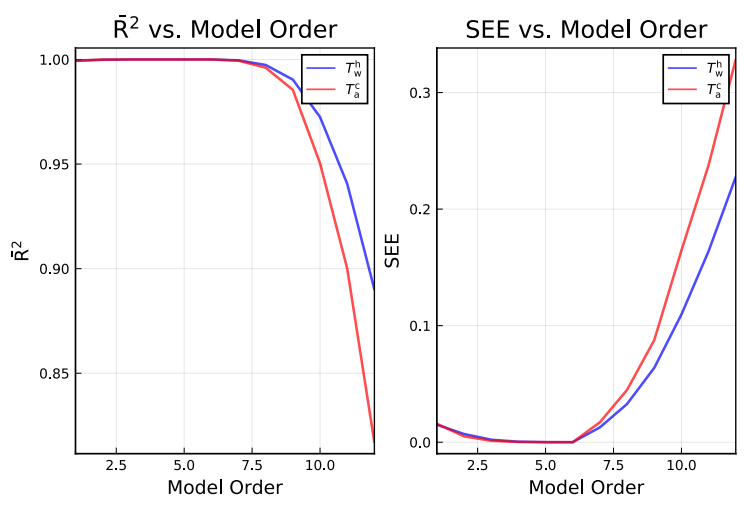

Figure 5. $\overline{\mathrm{R}}^{2}$ and SEE of case 1 . Left panel: The adjusted coefficient of determination vs. model order. Right panel: The standard error of estimate vs. model order.

However, the results so far are based on models that used the same data set for training and testing purposes, which is not a good indicator of the models' predictive ability. To evaluate a model's predictive performance and select the best fit model, validation is required.

In this work, the Holdout method was implemented to validate the models. The holdout method is one of the simplest cross-validation methods, where the data is split randomly into two data sets; a training set to estimate the model's parameters, and a test set to assess the performance of the model. The training data set is usually larger
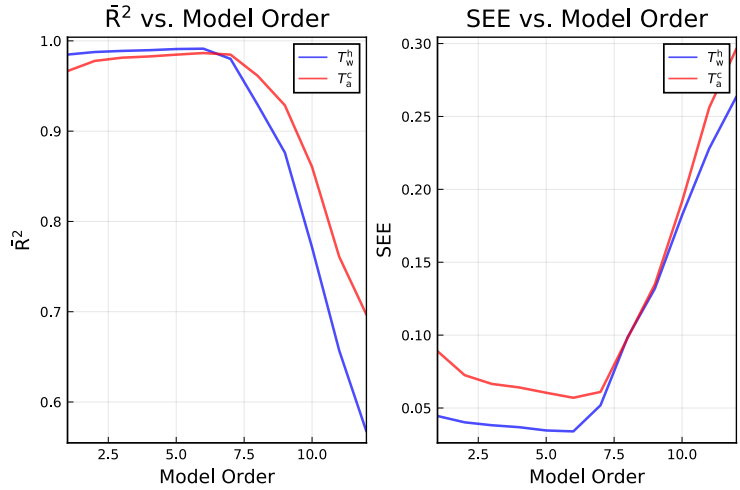

Figure 6. $\overline{\mathrm{R}}^{2}$ and SEE of case 2 . Left panel: The adjusted coefficient of determination vs. model order. Right panel: The standard error of estimate vs. model order.
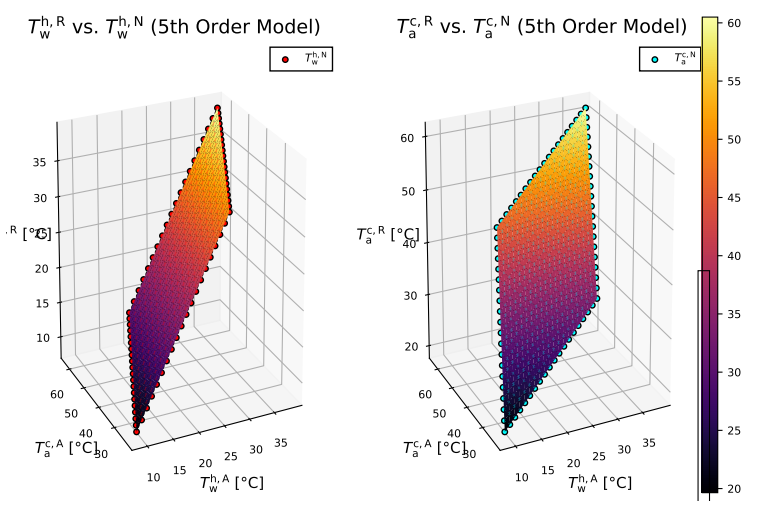

Figure 7. Case 1: A comparison between the regression model (the surfaces in the figure) and the numerical solution of the nonlinear two-point boundary value problem (the data points) for a 5 th order regression model.

than the test set. Typically, the holdout method involves a single run. In this work, the results of multiple runs are averaged together to avoid misleading results. The validation results are presented in Table 4 for case 1 , and in Table 5 for case $2 .^{6}$

Finally, the best fit model of case 1 achieved an average RMSE of $7 \cdot 10^{-8}{ }^{\circ} \mathrm{C}$ and $2 \cdot 10^{-8}{ }^{\circ} \mathrm{C}$ for $T_{\mathrm{w}}^{\mathrm{h}}$ and $T_{\mathrm{a}}^{\mathrm{c}}$, respectively. Also, the best fit model of case 2 achieved an average RMSE of $0.117^{\circ} \mathrm{C}$ and $0.327^{\circ} \mathrm{C}$ for $T_{\mathrm{w}}^{\mathrm{h}}$ and $T_{\mathrm{a}}^{\mathrm{c}}$, respectively.

\section{Nonlinear regression}

Nonlinear regression of the counter-current heat exchanger model is implemented in Julia using the package Flux (Innes, 2018; Innes et al., 2018). Moreover, the nonlinear mapping between the analytic solution of the ideal counter-current heat exchanger model and the numeric solution of the non-ideal heat exchanger model (the case of temperature dependence in the specific heat capacities of air and water) is achieved using the logistic (also known

\footnotetext{
${ }^{6}$ Both SEE and $\overline{\mathrm{R}}^{2}$ are rounded to the 5 th digit after the decimal place.
} 

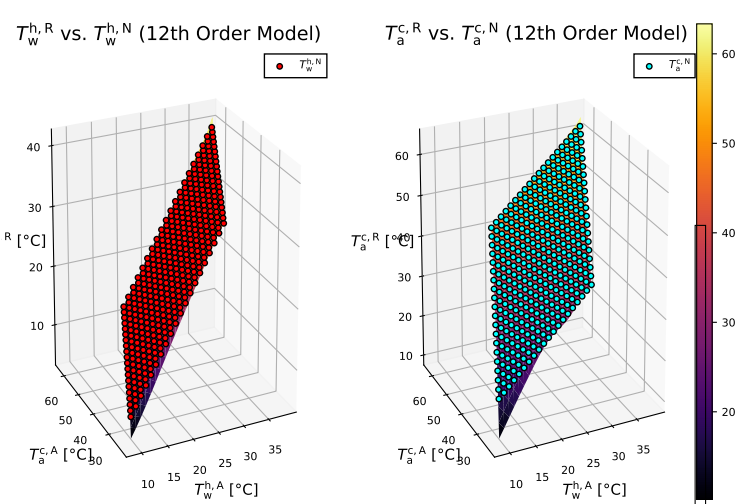

Figure 8. Case 1: A comparison between the regression model (the surfaces in the figure) and the numerical solution of the nonlinear two-point boundary value problem (the data points) for a 12th order regression model.
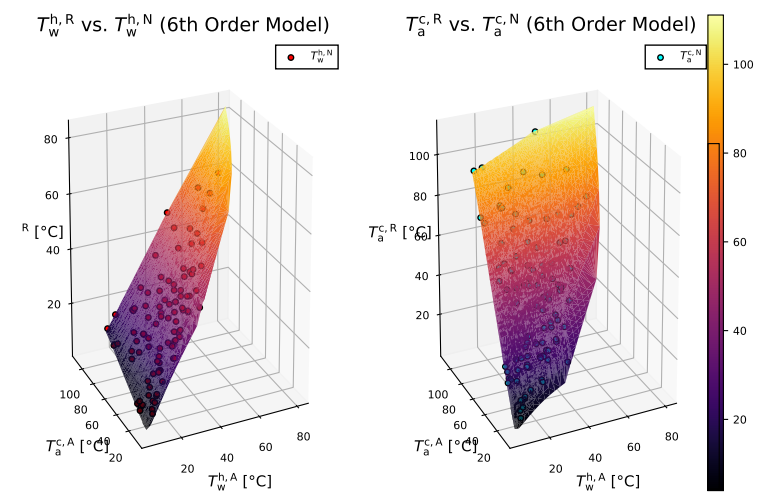

Figure 9. Case 2: A comparison between the regression model (the surfaces in the figure) and the numerical solution of the nonlinear two-point boundary value problem (the data points) for a 6 th order regression model.

as sigmoid) activation function $(\sigma)$, which is introduced between two linear layers in the classical Feedforward Neural Network (FNN). Furthermore, the FNN is implemented using the description in (Lie, 2019) and Flux documentation. In this work, the nonlinear regression model is composed of two dense layers with the non-linearity $(\sigma)$ between them as illustrated in Fig. 11.

In a similar manner to linear regression, the holdout method was implemented to select the dimension (out) in Fig. 11 that gives the best fit. The validation results are presented in Table 6 and Fig. 12, where RMSE was chosen as an indicator of goodness-of-fit. In Fig. 12, it is apparent that increasing the dimension (out) beyond 80 would result in a worse fit. Training of neural networks is sensitive to scaling of the data, and it is common practice to either normalize or standardize the data. Here, data $X$ and $Y$ have been normalized to $\tilde{X}$ and $\tilde{Y}$ in the range $[0,1]$, e.g.,

$$
\tilde{X}=\frac{X-X_{\min }}{X_{\max }-X_{\min }}
$$
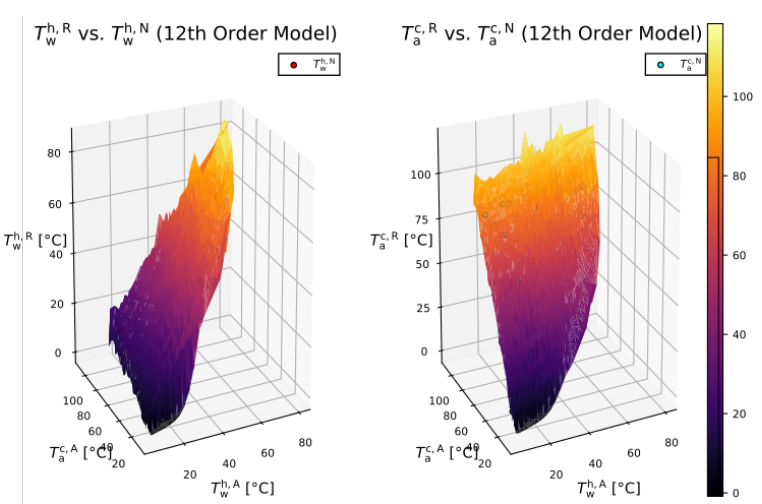

Figure 10. Case 2: A comparison between the regression model (the surfaces in the figure) and the numerical solution of the nonlinear two-point boundary value problem (the data points) for a 12 th order regression model.

Table 4. Validation results of case 1.

\begin{tabular}{c|cccc}
\multirow{2}{*}{$\begin{array}{c}\text { Model } \\
\text { order }\end{array}$} & \multicolumn{2}{|c}{ Avg. SEE, ${ }^{\circ} \mathrm{C}$} & \multicolumn{2}{c}{ Avg. $\overline{\mathrm{R}}^{2},-$} \\
\cline { 2 - 5 } & $T_{\mathrm{w}}^{\mathrm{h}}$ & $T_{\mathrm{a}}^{\mathrm{c}}$ & $T_{\mathrm{w}}^{\mathrm{h}}$ & $T_{\mathrm{a}}^{\mathrm{c}}$ \\
\hline \hline 1 & 0.01798 & 0.01905 & 0.99938 & 0.99944 \\
2 & 0.00858 & 0.0061 & 0.99986 & 0.99994 \\
3 & 0.00267 & 0.00149 & 0.99999 & 1.0 \\
4 & 0.00056 & 0.00028 & 1.0 & 1.0 \\
5 & $7 \cdot 10^{-5}$ & $4 \cdot 10^{-5}$ & 1 & 1 \\
6 & 0.0001 & 0.00012 & 1 & 1 \\
7 & 0.01823 & 0.02451 & 0.99947 & 0.99923 \\
8 & 0.04843 & 0.06575 & 0.99642 & 0.99474 \\
9 & 0.09783 & 0.13253 & 0.98625 & 0.97992 \\
10 & 0.17664 & 0.27147 & 0.95805 & 0.91988 \\
11 & 0.27841 & 0.4126 & 0.90428 & 0.832 \\
12 & 0.41207 & 0.59617 & 0.81076 & 0.68251 \\
\hline
\end{tabular}

\section{Results and Discussion}

In linear/nonlinear regression, the counter-current heat exchanger model was solved for a variety of conditions $\left(T_{\mathrm{w}}^{\mathrm{c}}\right.$, $T_{\mathrm{a}}^{\mathrm{h}}, \dot{m}_{\mathrm{w}}, \dot{m}_{\mathrm{a}}$, and thermal dependencies) to generate a data matrix. Then, validation was used to select the order of the polynomial and the dimension (out) that gives the best fit. Both case 2 of linear regression and nonlinear regression obtained a similar RMSE. Case 1 of linear regression achieved lower SEE and RMSE compared to case 2. However, case 1 is only valid for a constant mass flow rate, whereas case 2 is valid over a wide range of mass flow rates. This highlights the importance of selecting the appropriate ranges of $T_{\mathrm{w}}^{\mathrm{c}}, T_{\mathrm{a}}^{\mathrm{h}}, \dot{m}_{\mathrm{w}}$ and $\dot{m}_{\mathrm{a}}$ when solving the model. Selecting the appropriate ranges will result in a lower model order and a better fit.

In this work, the polynomial of the design matrix was generated using a user made function, which made the execution speed of the hybrid solution with linear regression relatively slower than the execution speed of the hybrid solution with nonlinear regression. Table 7 compares the execution speed of both solutions. To present a fair comparison between the linear and the nonlinear models, a 2nd 
Table 5. Validation results of case 2.

\begin{tabular}{c|cccc}
\multirow{2}{*}{$\begin{array}{c}\text { Model } \\
\text { order }\end{array}$} & \multicolumn{2}{|c}{ Avg. SEE, ${ }^{\circ} \mathrm{C}$} & \multicolumn{2}{c}{ Avg. $\overline{\mathrm{R}}^{2},-$} \\
\cline { 2 - 5 } & $T_{\mathrm{w}}^{\mathrm{h}}$ & $T_{\mathrm{a}}^{\mathrm{c}}$ & $T_{\mathrm{w}}^{\mathrm{h}}$ & $T_{\mathrm{a}}^{\mathrm{c}}$ \\
\hline \hline 1 & 0.0529 & 0.1059 & 0.9848 & 0.9665 \\
2 & 0.0479 & 0.0864 & 0.9876 & 0.9777 \\
3 & 0.0456 & 0.0795 & 0.9888 & 0.9812 \\
4 & 0.0443 & 0.0771 & 0.9895 & 0.9825 \\
5 & 0.0418 & 0.0731 & 0.9908 & 0.9844 \\
6 & 0.0415 & 0.0695 & 0.991 & 0.9862 \\
7 & 0.0686 & 0.0783 & 0.976 & 0.9828 \\
8 & 0.1246 & 0.1246 & 0.9241 & 0.9582 \\
9 & 0.1731 & 0.1774 & 0.8606 & 0.9192 \\
10 & 0.2467 & 0.2587 & 0.7357 & 0.8396 \\
11 & 0.3229 & 0.3556 & 0.5897 & 0.7253 \\
12 & 0.4014 & 0.4455 & 0.447 & 0.6229 \\
\hline
\end{tabular}

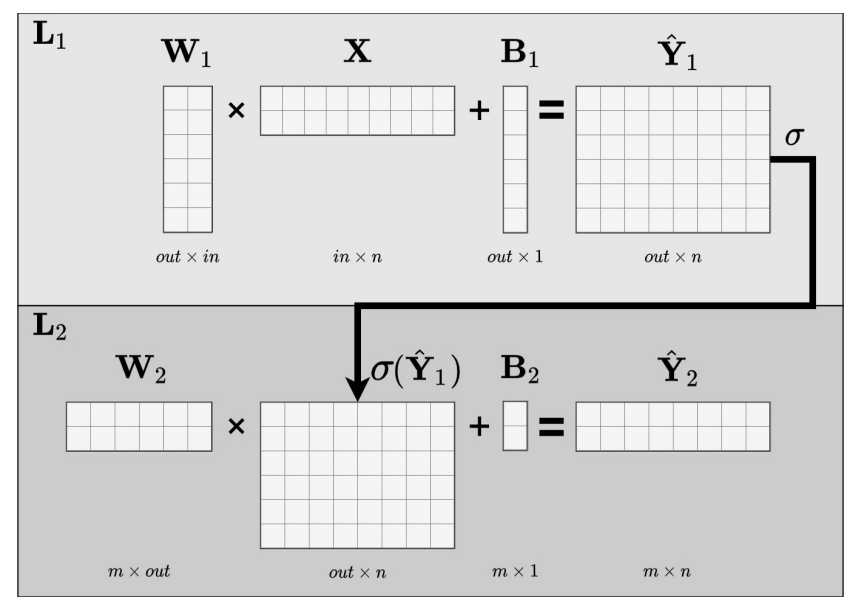

Figure 11. Feedforward neural network with two dense layers (L1 and L2) and an activation function $(\sigma)$ in between them.

order linear model was hard-coded in the design matrix, which also provides a good enough prediction accuracy.

The simulation time of the final hybrid models, the combination of the correction terms of linear/nonlinear regression with the analytic expressions of equations 1 and 2 , is presented in Table 8 for the heat exchanger model, and in Table 9 for the thermal model of an air-cooled synchronous generator. The hybrid solutions of the heat exchanger sub-model achieved similar execution speeds and are much faster than the numeric solution of the nonlinear two-point boundary value problem. Similarly, the simulation time of the thermal model of an air-cooled synchronous generator was significantly reduced by using the hybrid models.

\section{Conclusions}

In this paper, the thermal model of the counter-current heat exchanger that was developed in $\mathrm{Lie}^{7}$, is extended with the case of temperature dependence in the specific heat ca-

\footnotetext{
${ }^{7}$ Lie, B.: Lecture Notes in course FM1015 Modelling of Dynamic Systems at University of South-Eastern Norway, 2019.
}

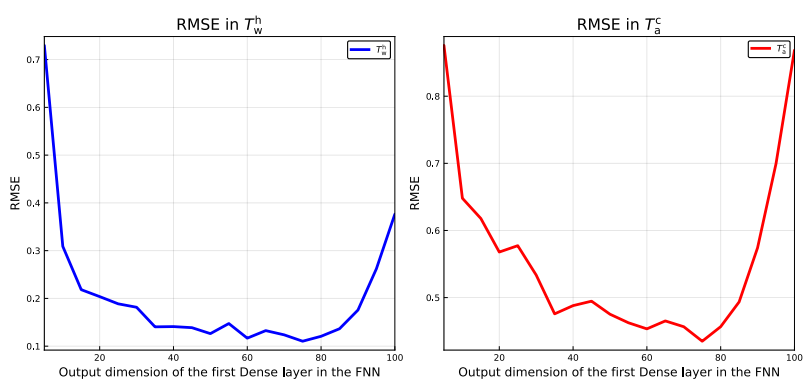

Figure 12. Validation results of nonlinear regression of the counter-current heat exchanger model. The Y-axis is the average RMSE for three models, each trained on a random sample for 10000 epoch. The best fit model achieved an average RMSE of $0.110^{\circ} \mathrm{C}$ and $0.435^{\circ} \mathrm{C}$ for $T_{\mathrm{w}}^{\mathrm{h}}$ and $T_{\mathrm{a}}^{\mathrm{c}}$, respectively.

Table 6. Validation results of nonlinear regression.

\begin{tabular}{c|cc} 
Model & \multicolumn{2}{|c}{ Avg. RMSE, ${ }^{\circ} \mathrm{C}$} \\
\cline { 2 - 3 } order & $T_{\mathrm{w}}^{\mathrm{h}}$ & $T_{\mathrm{a}}^{\mathrm{c}}$ \\
\hline \hline 5 & 0.7297 & 0.8757 \\
10 & 0.309 & 0.648 \\
15 & 0.2183 & 0.6179 \\
20 & 0.2038 & 0.5679 \\
25 & 0.1887 & 0.5774 \\
30 & 0.1815 & 0.5336 \\
35 & 0.1405 & 0.4758 \\
40 & 0.141 & 0.4881 \\
45 & 0.1387 & 0.4947 \\
50 & 0.1263 & 0.4752 \\
55 & 0.1474 & 0.4625 \\
60 & 0.1168 & 0.4534 \\
65 & 0.1326 & 0.4652 \\
70 & 0.1237 & 0.4566 \\
75 & 0.1104 & 0.435 \\
80 & 0.1207 & 0.4567 \\
85 & 0.1365 & 0.4936 \\
90 & 0.1756 & 0.5742 \\
95 & 0.2621 & 0.6995 \\
100 & 0.3765 & 0.8686 \\
\hline
\end{tabular}

pacities of air and water. The benchmark results showed a very long simulation time when solving the nonlinear boundary value problem numerically. To speed up the solution time, explicit data-driven models were developed using linear and nonlinear regression. Validation was used to select the order of the polynomial of the design matrix and the dimension of the layers in the FNN. However, the order of the polynomial and the dimension of the layers were not the only factors that impacts the accuracy of the models. The accuracy of the models was also affected by the generated data matrix on which the regression models were fitted. The generated data matrix should only contain informative data that relates to the model's objective. Moreover, the regression models were expressed as a correction term to the explicit/analytic ideal heat exchanger model. The hybrid heat exchanger model achieved a good enough accuracy and faster execution speed compared to 
Table 7. The execution speed of the hybrid solution: linear regression vs nonlinear regression. Here, the polynomial of the design matrix was generated using a user made function, which made this comparison not fair. Linear-hybrid model has mean time $36 \mu$ s on a given computer; times are scaled to unity at $1^{\text {st }}$ row element.

\begin{tabular}{lc}
\hline Model & Mean time \\
\hline Hybrid solution (Linear Reg.) & 1 \\
Hybrid solution (Nonlinear Reg.) & 0.042 \\
\hline
\end{tabular}

Table 8. Simulation time for the heat exchanger model. Linearhybrid model has mean time $1.5 \mu \mathrm{s}$ on a given computer; times are scaled to unity for $1^{\text {st }}$ row element.

\begin{tabular}{lc}
\hline Model & Mean time \\
\hline Hybrid solution (Linear Reg.) & 1 \\
Hybrid solution (Nonlinear Reg.) & 1.002 \\
Numeric solution & 14070 \\
\hline
\end{tabular}

the numeric solution.

It should be observed that the hybrid heat exchanger model introduced here is based upon the assumption that there are no unknown model parameters in the heat exchanger. In other words: if we want to include the hybrid heat exchanger model in a model fitting procedure for the thermal generator model, we can not change the heat exchanger parameters, e.g., $\mathscr{U} A_{\mathrm{x}}$ in Table 1 - that would require a re-training of the correction term.

Future work will include a study on how the correction term to the analytic heat exchanger can be re-formulated in order to allow for fitting model parameters such as $\mathscr{U} A_{\mathrm{x}}$ without having to re-train the correction term. As part of this, the value of $\mathscr{U}_{\mathrm{x}}$ will vary with flow rates. Next, the work reported in (Pandey et al., 2019) will be updated with this new thermal synchronous generator model with the more accurate heat exchanger model.

\section{References}

Damodar N. Gujarati. Linear Regression: A Mathematical Introduction. SAGE Publications, Inc, 2019. ISBN 978-1-54433657-2. doi:10.4135/9781071802571.

Frank P. Incropera, David P. Dewitt, Theodore L. Bergman, and Adrienne S. Lavine. Principles of Heat and Mass Transfer. International Student Version. John Wiley \& Sons, Inc., Singapore, 7 edition, 2013. ISBN 968-0-470-64615-1.

Michael Innes, Elliot Saba, Keno Fischer, Dhairya Gandhi, Marco Concetto Rudilosso, Neethu Mariya Joy, Tejan Karmali, Avik Pal, and Viral Shah. Fashionable modelling with flux. CoRR, abs/1811.01457, 2018. doi:1811.01457.

Mike Innes. Flux: Elegant Machine Learning with Julia. Journal of Open Source Software, 2018. doi:10.21105/joss.00602.

Bernt Lie. Surrogate and hybrid models for control. In Proceedings of the 60th Conference on Simulation and Modelling, volume 170 of Linköping Electronic Conference Proceedings, pages 1-8, University of Västerås, Västerås, Sweden,
Table 9. Simulation time for the thermal model of an air-cooled synchronous generator. Linear-hybrid model has mean time $2.5 \mathrm{~ms}$ on a given computer; times are scaled to unity for $1^{\text {st }}$ row element.

\begin{tabular}{lc}
\hline Model & Mean time \\
\hline Hybrid solution (Linear Reg.) & 1 \\
Hybrid solution (Nonlinear Reg.) & 1.1 \\
Numeric solution & 3920 \\
\hline
\end{tabular}

August 2019. SIMS, Linköping University Electronic Press. doi:https://doi.org/10.3384/ecp201701.

Bonnie J McBride, Michael J Zehe, and Sanford Gordon. NASA Glenn Coefficients for Calculating Thermodynamic Properties of Individual Species. Technical Report NASA/TP2002-21155, NASA, NASA Center for Aerospace Information 7121 Standard Drive Hanover, MD 21076, 2002. URL http://gltrs.grc.nasa.gov/GLTRS.

Kathleen E. Murphy. Thermodynamics Problem Solving in Physical Chemistry: Study Guide and Map. CRC Press, 2020. ISBN 978-1-00-003028-0.

Thomas Øyvang. Enhanced power capability of generator unites for increased operational security. $\mathrm{PhD}$ thesis, University of South-Eastern Norway, Faculty of Technology, Natural Sciences and Maritime Sciences University of SouthEastern Norway N-2018 Porsgrunn Norway, December 2018. ISBN: 978-82-7206-503-3 (print) ISBN: 978-82-7206-504-0 (online).

Madhusudhan Pandey. Model fitting and state estimation for thermal model of synchronous generator. mathesis, University of South-Eastern Norway, Porsgrunn, Norway, 2019.

Madhusudhan Pandey, Thomas Øyvang, and Bernt Lie. State estimation of a thermal model of air-cooled synchronous generator. In Proceedings of the 60th Conference on Simulation and Modelling, volume 170 of Linköping Electronic Conference Proceedings, pages 190-197, University of Västerås, Västerås, Sweden, August 2019. SIMS, Linköping University Electronic Press. doi:https://doi.org/10.3384/ecp20170190.

Christopher Rackauckas and Qing Nie. DifferentialEquations.jl - A Performant and Feature-Rich Ecosystem for Solving Differential Equations in Julia. Journal of Open Research Software, 5(15), 2017. doi:10.5334/jors.151.

Gary Smith. Essential Statistics, Regression, and Econometrics, chapter 10 - Multiple Regression, pages 301-337. Academic Press, second edition, 2015. doi:https://doi.org/10.1016/B978-0-12-803459-0.000108 . 